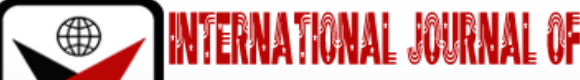

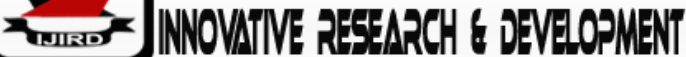

ISSN 2278 - 0211 (Online)

\section{Design and Implementation of an Emergency Traffic Light Pre-emption System Using PLC Automation}

Kofi Addo Annan
Research Assistant, Department of Electrical/Electronic Engineering,
Kwame Nkrumah University of Science and Technology, Ghana
Emmanuel Obuobi Kwame Addo
Lecturer, Department of Electrical/Electronic Engineering,
Kwame Nkrumah University of Science and Technology, Ghana
Jephthah Afedzi Baidoo
Electrical Engineer Graduate Trainee, Department of Engineering,
Process and Plant Automation Limited, Ghana
Kwame Opare-Anim
Electrical Engineer Graduate Trainee, Department of Engineering
HUAWEI, Ghana

\section{Abstract:}

As traffic on the urban Ghanaian road network increases, congestion and delays are becoming more severe. Traffic signals are the most common method by which right of way is dynamically allocated to conflicting movements. Ghanaian traffic light control system is mainly designed by using devices such as timers, relays etc. The critical timing operation is required to be carried out under the existence of heavy traffic situations. This does not give this conventional traffic light control system the flexibility to control throughput to favor emergency vehicles. This conventional practice leads to many problems and subsequent delay for a long time. With the help of a PLC, the requirement of fast automation and effective optimization of traffic light control even in times of emergency can be achieved.

At intersections, capacity bottlenecks are formed in urban road networks because at these locations, capacity must be shared by competing traffic movements. For this reason, this thesis introduces an execution and implementation of T-junction emergency traffic control system using SEIMENS S7-200 PLC. Programming in PLC is written in ladder logic with the help of STEP7 MICROWIN software.

Keywords: Programmable logic controller (PLC), T-junction traffic light control

\section{Introduction}

The traffic light, also known as traffic signal, stop light, traffic lamp, stop-and-go lights is a signaling device positioned intersection pedestrian crossing, or other location. Its purpose is to indicate, using a series of colors (Red Amber - Green), the correct moment to stop, drive, ride or walk, (using a universal color code and a precise sequence, for those who are color blind) (Sharma \& Sivani, 2014). A traffic signal is an electronic device that assigns right-of-way at an intersection or street crossing by means of displaying the standard red, yellow, and green-colored indications. The use of these colors is thought to originate from nautical right-of -way.

Achieving safe and less delays in transportation was a major reason for the development of the traffic signal system to manage and control conflicts on the use of intersections on our road networks. Even with the introduction of the traffic light, it was not enough to reduce the delays by emergency vehicles when responding to emergencies such as medical needs and fire response, with the increase in population, the need for a much faster response time for emergency vehicle became necessary. Using the traffic signal to achieve this meant developing a traffic signal system that automatically give the right of way to emergency vehicles then reverts to normal signal control in the absence of emergency vehicles. Signal preemption technology is a unique effort used by emergency vehicles such as fire engines ambulances and police cars in satisfying the growing demand of emergency response system. Some regions have signals that are interruptible, giving priority to special traffic. Such traffic light preemption is usually reserved for emergency vehicles such as fire engines, ambulances and police squad cars, though sometimes presidential and other VIP convoys can interrupt lights. Upon activation, the normal traffic light cycle is suspended and replaced by the 'preemption sequence'. Here the traffic light to all approaches to the intersection are switched to 'red' with the exception of the light for the vehicle that triggered the sequence. (Sharma \& Sivani, 2014) 


\section{Types of Traffic Signal Operations}

\subsection{Fixed-Time Mode}

Under this mode, there are no detections for any approach. The signal continuously cycles regardless of actual traffic demand. Pedestrian walk signals are automatic and will cycle concurrently with the vehicular signal indication. (Qi, Zhou, \& Luan, 2015)

The simplest control system uses a timer. Each phase of the signal lasts a specific duration before the next phase occurs. They however have the major disadvantage when the signal timing of an intersection will bebetter adapted to the dominant flows changing throughout the day.

\subsection{Semi-Actuated Mode}

Under this mode, the detection system is present only on a minor cross street. When detection is activated, the green light on a major street is interrupted to allow the minor street traffic and pedestrians to safely enter the intersection. Pedestrian walk signals for crossing a minor street are automatic, while those for crossing a major street are not. Pedestrians crossing a major street must push the 'pedestrian push button' to get the walk signal.(Zald'ıvar-Colado et al., 2017)

\subsection{Actuated Mode}

Some traffic lights at pedestrian crossings, especially those away from junctions, includea button, which must be pressed in order to activate the timing system. This is usually accompanied by alarge display, which reads, 'wait' that lights up when the button is pressed. The traffic signal is set to provide the green light 'on-demand' or only in the presence of vehicles. Pedestrians MUST push the pedestrian push button in order to cross either the major or the minor streets. (Kissell, Karr, \& Overstreet,2003)

\subsection{Dynamic Control}

More sophisticated control systems use electronic detector loops, which are sensors buried in the pavement or over-roadway sensors to detect the presence of traffic waiting at the light, and thus can avoid giving the green light to an empty road while motorists on a different route are stopped. A timer is frequently used as a backup in case the sensors fail; an additional problem with sensor-based systems is that they may fail to detect vehicles such as motorcycles or bicycles and cause them to wait indefinitely (or at least until a detectable vehicle also comes to wait for the light). The sensor loops typically work in the same fashion as metal detectors; small vehicles or those with low metal content may fail to be detected.(Bonner \& Faerber, 1980)

In order for efficient use of traffic signal operations, a traffic management system is used. Traffic management systems are computerized systems that control traffic signal systems. The computer monitors the traffic signal throughout the day. The information from the system, allows city traffic engineers to make signal timing adjustments. Technological applications in traffic engineering are called INTELIGENT TRANSPORTATION SYSTEMS (ITS). Some of these devices include:

- $\quad$ Closed Circuit Television cameras (CCTV)

- $\quad$ Changeable Message Signs (CMS)

These signs display useful information to alert motorists of road conditions ahead during event days. (Mitchell,

1984)

- $\quad$ Programmable Visibility Signals

Using special adhesive tapes and programming lenses, signals are masked so that only certain lanes of traffic can view indication.

\subsubsection{Conventional Lighting Systems}

Conventional traffic signal lighting, still common in some areas, utilizes a standard light bulb typically; a 67 watt, 69 watt, or 115 watt medium-base (household lamp) light bulb provides the illumination. Light then bounces off a mirrored glass or polished aluminum reflector bowl, and out through a polycarbonate plastic or glass signal lens. In some signals, these lenses were cut to include a specific refracting pattern. (Hashim et al., 2013)

However, the traditional bulb is being phased out. In newer lighting systems, LED arrays are used. Their longer life span (about two years) and higher efficiency makes them a better choice. It is noted however that thanks to all the excess heat generated by the incandescent bulbs, traditional traffic lights were able to weather even the worst of snow and ice storms, melting off any accumulation that would threaten to obscure the view. LEDs, owing to their greater efficiency, were not. The solution for this problem was built-in heating modules with the LED arrays. (Mitchell, 1984)

\section{Methodologies}

From the literatures, we can conclude that full automation is required for making smart traffic control system, which can take care for the emergency vehicles. In this paper, the primary aim is to design technically the automation of a T-junction. Considering the T-junction to be the basic infrastructural element of the whole Ghanaian road system, the emergency traffic signal system should be able to do the following:

- Intelligent traffic signal system based on the volume of traffic on each side of the signal.

Optimize the following:

- $\quad$ Minimize the average waiting time of emergency vehicles. 
- $\quad$ First, come first serve to all emergency vehicles except ambulances and fire service trucks.

- Give ambulance and fire service trucks priority over all other vehicles.

- Minimizing the number of accidents that can occur during emergency phase.

\subsection{T-Junction Traffic Control System}

The model view of a T-junction traffic control system is shown below. It is simulated with the WINCC software, which allows for STEP 7 integration. It is simulated with four lanes lane 1, lane 2, lane 3 and lane4. Each lane consists of three lights red, green and an emergency blue flashing light.

Top View of a T-Junction with Traffic Lights

Totally it requires two input switches; an 'On' and an 'Off' switch with the following port numbers.

\begin{tabular}{|c|c|}
\hline OFF BUTTON & I 0.0 \\
\hline ON BUTTON & I 0.5 \\
\hline \multicolumn{2}{|c}{ Table 1}
\end{tabular}

And twelve outputs each with the following ports.

\begin{tabular}{|c|c|}
\hline EMERGENCY LIGHT 1 & Q 1.1 \\
\hline EMERGENCY LIGHT 2 & Q 1.2 \\
\hline EMERGENCY LIGHT 3 & Q 1.3 \\
\hline EMERGENCY LIGHT 4 & Q 1.4 \\
\hline GREEN 1 & Q 0.1 \\
\hline GREEN 2 & Q 0.2 \\
\hline GREEN 3 & Q 0.3 \\
\hline GREEN 4 & Q 0.4 \\
\hline RED 1 & Q 0.5 \\
\hline RED 2 & Q 0.6 \\
\hline RED 3 & Q 0.7 \\
\hline RED 4 & Q 1.0 \\
\hline
\end{tabular}

Table 2: Top View of a T-Junction with Traffic Lights

The total operation of this system takes place in sequence of operation of states, which are explained below.

\subsection{Sequence of Operation}

The operation of the T-junction traffic control system takes place in two main stages each with a sequence of operation of states. The two stage are;

- Traffic control during the normal Phase (no emergency vehicles)

- Traffic control during emergency phase.

Traffic Control during the Normal Phase (No Emergency Vehicles)

During this stage, traffic control is obtained by only timer control relays of the PLC. There are four states in the operation;

- State 1: In this state the traffic of lane, one is allowed and the traffic of lane two, three and four are stopped. Here in this state, green light of lane one (G1) glows and red lights of lane two, three and four (R2), (R3) and (R4) glows. This period is continued for fifteen-second duration.

- State 2: In this state, the traffic of lane two is allowed and the traffic of lane one, three and four are stopped. Here in this state, green light of lane two (G2) glows and red lights of lane one, three and four (R1), (R3) and (R4) glows. This period is continued for fifteen-second duration.

- State 3: In this state, the traffic of lane three is allowed and the traffic of lane one, two and four are stopped. Here in this state, green light of lane three (G3) glows and red lights of lane one, two and four (R1), (R2) and (R4) glows. This period is continued for fifteen-second duration.

- $\quad$ State 4: In this state, the traffic of lane four is allowed and the traffic of lane one, two and three are stopped. Here in this state, green light of lane four (G4) glows and red lights of lane one, two and three (R1), (R2) and (R3) glows. This period is continued for fifteen-second duration.

Between each state, a gap of two seconds is left in order to prevent any collisions. After the execution of three stages is completed, the timer resets and the sequence of operation again start and it repeats continuously. During this period the emergency lights do not come on.

\subsubsection{Traffic Control during the Emergency Phase}

The emergency phase is only initiated when an emergency vehicle enters the vicinity of the T-junction. To control traffic during this stage, a combination of RF transmitters and receivers and timer control relays of the PLC are used to control the traffic lights. There are four states in the operation of the Traffic control during emergency phase.

- State 1: In this state, RF receiver on lane 1 detects the presence of an oncoming emergency vehicle on the lane with the help of the RF transmitter on top of the vehicle. The traffic of lane one is allowed and the traffic of lane 
two, three and four are stopped. Here in this state, green light of lane one (G1) glows, the Emergency Light (EM1) blinks and the red lights of lane two, three and four (R2), (R3) and

(R4) glows. This period is continued for fifteen seconds after the receiver stops receiving transmissions.

- State 2: In this state, RF receiver on lane 2 detects the presence of an oncoming emergency vehicle on the lane with the help of the RF transmitter on top of the vehicle. The traffic of lane two is allowed and the traffic of lane one, three and four are stopped. Here in this state, green light of lane two (G2) glows, the Emergency Light (EM2) blinks and the red lights of lane one, three and four (R1), (R3) and

(R4) glows. This period is continued for fifteen seconds after the receiver stops receiving transmissions.

- State 3: In this state, RF receiver on lane 3 detects the presence of an oncoming emergency vehicle on the lane with the help of the RF transmitter on top of the vehicle. The traffic of lane three is allowed and the traffic of lane one, two and four are stopped. Here in this state, green light of lane three (G3) glows, the Emergency Light (EM3) blinks and the red lights of lane one, two and four (R1), (R2) and

(R4) glows. This period is continued for fifteen seconds after the receiver stops receiving transmissions.

- State 4: In this state, RF receiver on lane 4 detects the presence of an oncoming emergency vehicle on the lane with the help of the RF transmitter on top of the vehicle. The traffic of lane four is allowed and the traffic of lane one, two and three are stopped. Here in this state, green light of lane four (G4) glows, the Emergency Light (EM4) blinks and red lights of lane one, two and three (R1), (R2) and

(R3) glows. This period is continued for fifteen seconds after the receiver stops receiving transmissions.

\subsection{State Table}

\begin{tabular}{|c|c|c|c|c|c|c|c|c|c|c|c|c|}
\hline State & G1 & R1 & EM1 & G2 & R2 & EM2 & G3 & R3 & EM3 & G4 & R4 & EM4 \\
\hline 1 & 1 & 0 & 0 & 0 & 1 & 0 & 0 & 1 & 0 & 0 & 1 & 0 \\
\hline 2 & 0 & 1 & 0 & 1 & 0 & 0 & 0 & 1 & 0 & 0 & 1 & 0 \\
\hline 3 & 0 & 1 & 0 & 0 & 1 & 0 & 1 & 0 & 0 & 0 & 1 & 0 \\
\hline 4 & 0 & 1 & 0 & 0 & 1 & 0 & 0 & 1 & 0 & 1 & 0 & 0 \\
\hline
\end{tabular}

Table 3: State Table for Traffic Control during the Normal Phase (No Emergency Vehicles)

\begin{tabular}{|c|c|c|c|c|c|c|c|c|c|c|c|c|c|c|c|c|}
\hline State & G1 & R1 & EM1 & G2 & R2 & EM2 & G3 & R3 & EM3 & G4 & R4 & EM4 & RF1 & RF2 & RF3 & RF4 \\
\hline 1 & 1 & 0 & 1 & 0 & 1 & 0 & 0 & 1 & 0 & 0 & 1 & 0 & 1 & 0 & 0 & 0 \\
\hline 2 & 0 & 1 & 0 & 1 & 0 & 1 & 0 & 1 & 0 & 0 & 1 & 0 & 0 & 1 & 0 & 0 \\
\hline 3 & 0 & 1 & 0 & 0 & 1 & 0 & 1 & 0 & 1 & 0 & 1 & 0 & 0 & 0 & 1 & 0 \\
\hline 4 & 0 & 1 & 0 & 0 & 1 & 0 & 0 & 1 & 0 & 1 & 0 & 1 & 0 & 0 & 0 & 1 \\
\hline
\end{tabular}

Table 4: State Table for Traffic Control during the Emergency Phase

\subsection{Flow Charts}

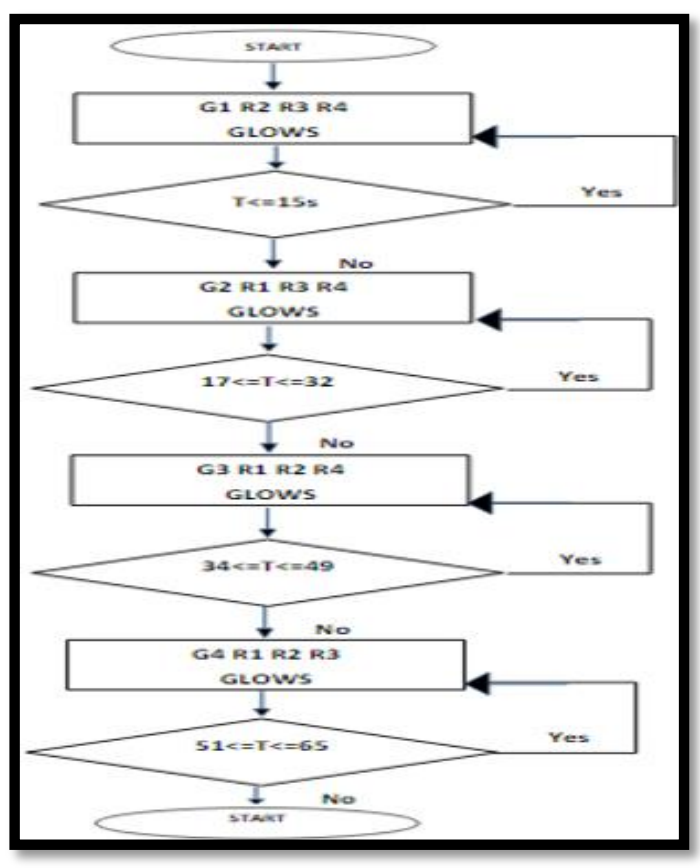

Figure 1: Flowchart Showing the Sequence of Operation of T-Junction Traffic Light Control System

In the given Figure 1 and 3 the flow charts represents the sequence of operation of T-junction traffic light control system in the normal state and emergency state respectively. 


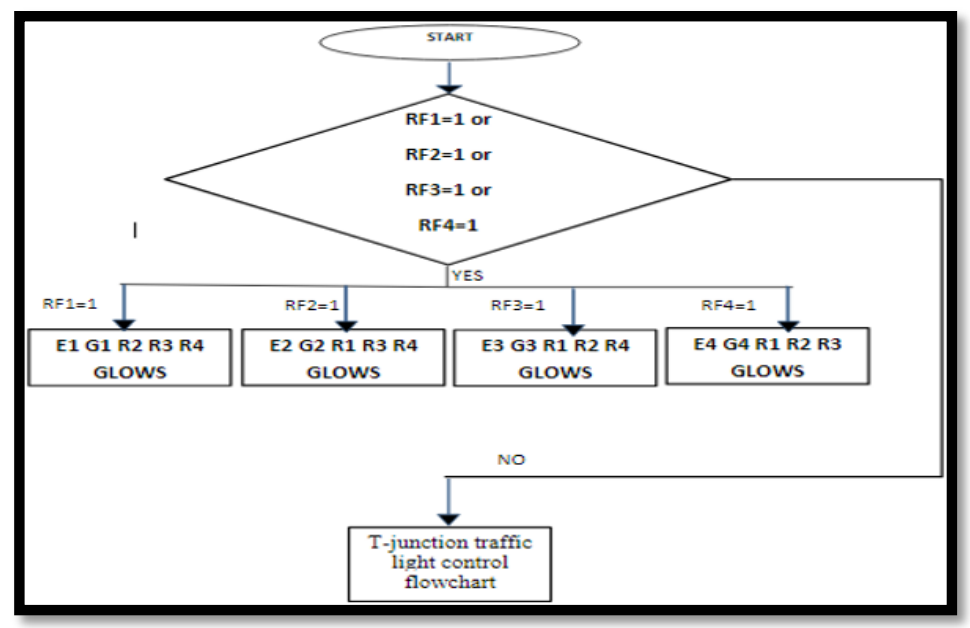

Figure 2: Flowchart Showing the Sequence of Operation of T-Junction Traffic Light Control System during Emergency Phase

\section{Design Considerations}

Based on the reviews, we came up with our design of our traffic system which will consist of three basic units;

- Transmitter Circuit

- Receiver Circuit

- Traffic Light Circuit

Basically, the traffic light pre-emption system design consists of the following electrical components;

- Programmable Logic Controller (PLC)

- 434MHz Transmitter and Receiver Pair

- Encoder-Decoder Pair

- LEDS

- Relays

- Breadboard Power Supplies

- Resistors

- Push Button Switches

\subsection{Programmable Logic Controller Device}

The PLC will be the brain of the system. It will control the normal operation of the traffic light. It will also provide the emergency mode controls once the emergency vehicle is detected. For this project, our requirements for the PLC were;

- At least 4 digital inputs.

- At least 5 digital outputs.

- A DC voltage output.

- A reasonable price of not more than US\$500.

- A readily available and easy to use software for programming the PLC.

- A very fast response.

- Enough memory to handle program.

Two PLC models currently sold in the market were compared. They were the;

- Siemens 1200 series with the $1214 \mathrm{CPU}$.

- Allen Bradley MicroLogix 1500 CPU

\begin{tabular}{|c|c|c|}
\hline PLC Model & Siemens S7-1214c & Micrologix1500 \\
\hline Input Voltage & $24 \mathrm{~V}$ DC & $20.4-26.4 \mathrm{~V}$ DC \\
\hline Output Voltage & $24 \mathrm{~V}$ DC & $24 \mathrm{~V}$ DC \\
\hline Number of Digital Inputs & 14 & 14 \\
\hline Number of Analog Inputs & 2 & 2 \\
\hline Number of Outputs & 10 & 14 \\
\hline Price & US 417 & US 591 \\
\hline Memory & $50 \mathrm{~kb}$ & $14 \mathrm{~kb}$ \\
\hline
\end{tabular}

Table 5: Comparison between the Two PLC Models

The PLC models available were equally good. The model S7-1214c was selected for this project because itmet the requirements at a cheaper price whilst giving larger memory space. 


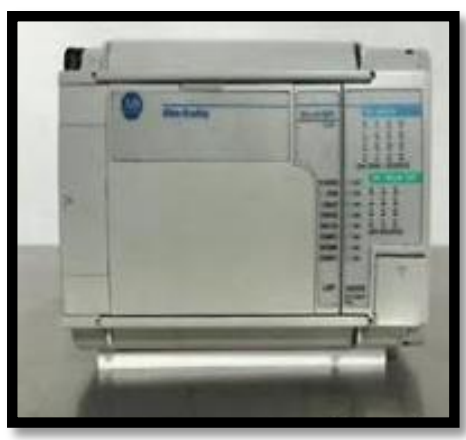

Figure 3: MicroLogix1500

\subsection{Selection of Transmitter-Receiver Pair}

The transmitter will be installed in the vehicle with the receiver installed on the traffic light. Once triggered, a signal will be sent from the emergency vehicle to the traffic controller, informing the controller of its presence. The requirements for the transmitter-receiver pair based on our project were;

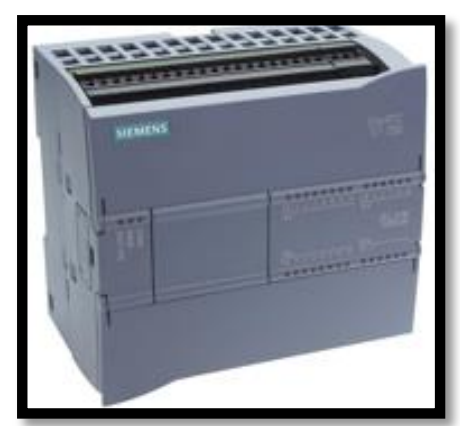

Figure 4: Siemens S7-1214C

- $\quad$ Should be reliable.

- $\quad$ Should have a good working range $(0-200 \mathrm{~m})$.

- $\quad$ Should be compatible with ICs to enable encoding and decoding.

- Should work on 5V DC.

\begin{tabular}{|c|c|c|}
\hline RF Transmitter Model & $\mathbf{8 6 8} \mathbf{~ M H z}$ & $\mathbf{4 3 4} \mathbf{~ M H z}$ \\
\hline Maximum Range & $160 \mathrm{~m}$ & $240 \mathrm{~m}$ \\
\hline Operating Voltage & $5 \mathrm{~V}$ & $5-12 \mathrm{~V}$ \\
\hline
\end{tabular}

Table 6: Comparison between Transmitter-Receiver Pair

The $434 \mathrm{MHz}$ transmitter-receiver module was used because of it met our requirements and has greater range.

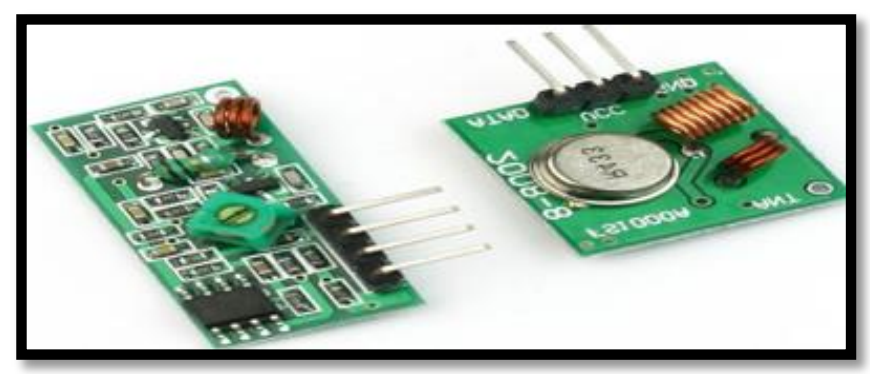

Figure 5: The 434 MHZ Transmitter-Receiver Module

\subsection{Encoder-Decoder Pair}

Data should be encoded before it is transmitted over a wireless link to ensure reliability and reduce the effects of interference and cross-talk. The requirements for the encoder-decoder pair for our project were;

- It should be capable of providing multiple channels for the 4 individual lanes.

- It should have a form of Parallel to Serial conversion system. 


\begin{tabular}{|c|c|c|}
\hline Encoder/Decoder Model & H12E & HC148 \\
\hline Input Voltage & $2.4-12$ V DC & 2-6V DC \\
\hline Specialty & Parallel to serial connection & Priority Encoder \\
\hline Number of Address Pins & 8 & 8 \\
\hline Number of Data Pins & 12 & 12 \\
\hline Number of Words Sent Per Time & 4 & 4 \\
\hline Price & US\$1.34 & US \$5.06 \\
\hline
\end{tabular}

Table 7: Comparison between Encoder-Decoder Pair

There was no need to prioritize any of the inputs since the emergency system works on a first come first serve basis with each lane having equal priority. Thus the H12 Encoder/Decoder pair was selected.

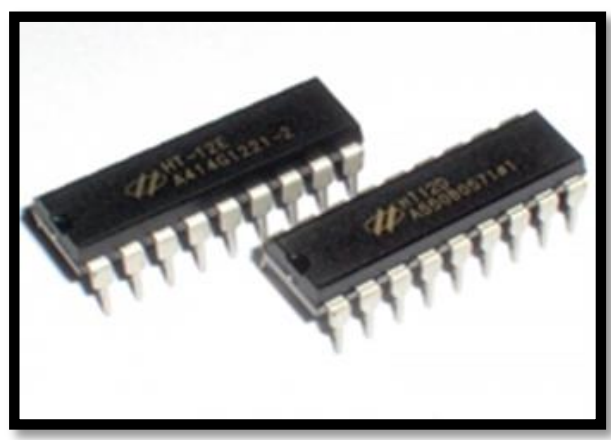

Figure 6: Encoder-Decoder Pair

\section{Design Implementation}

The transmitter and receiver were connected as shown below. The receiver circuit is connected straight into the PLC as shown above. The transmitter and receiver are powered with a bread board power supply. The power supply provides a stable $5 \mathrm{~V}$ DC.

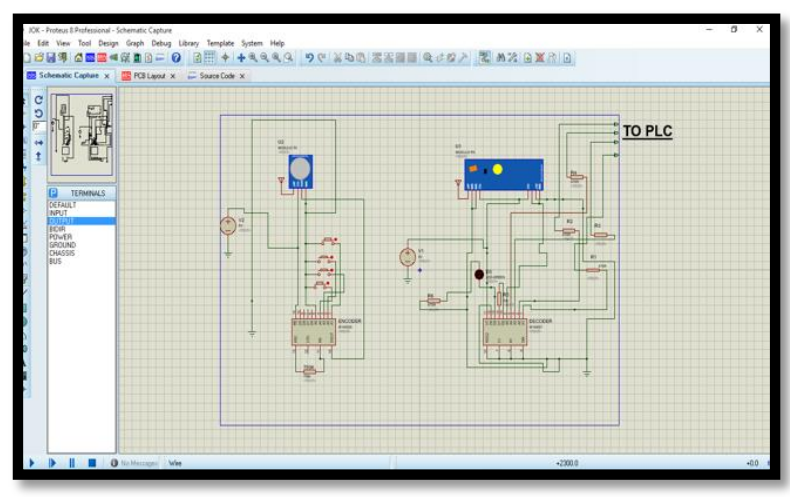

Figure 7: Shows the Transmitter-Receiver Circuit Simulated Using Proteus 8 Professional

The following figures $\mathrm{i}$, ii, iii, iv and v shows the program is uploaded into the PLC. The program was written using TIA PORTAL S7-PLCSIM V-14;

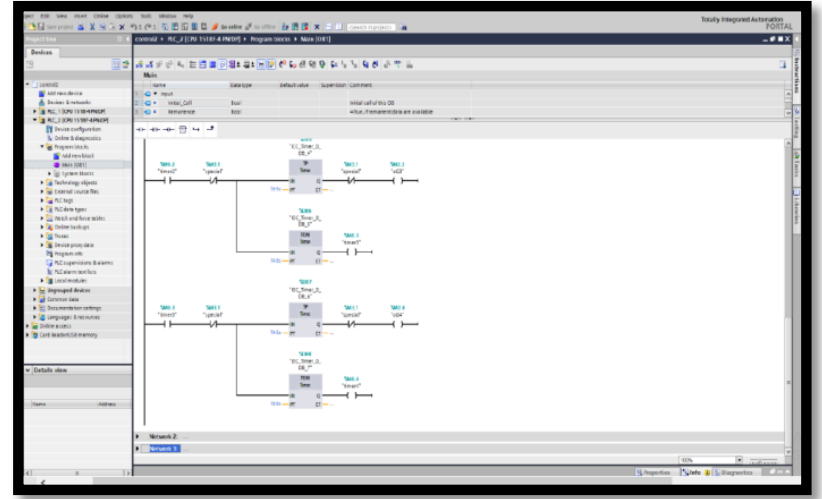

Figure 8 


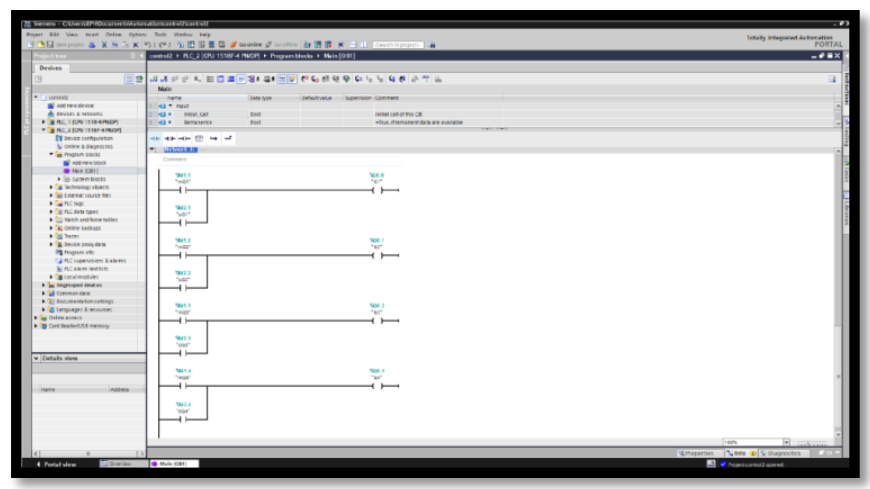

Figure 9

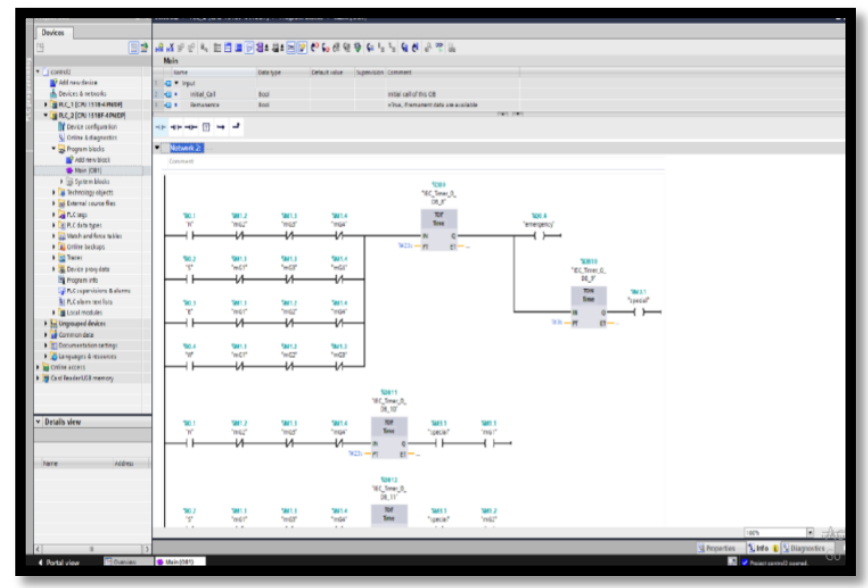

Figure 10

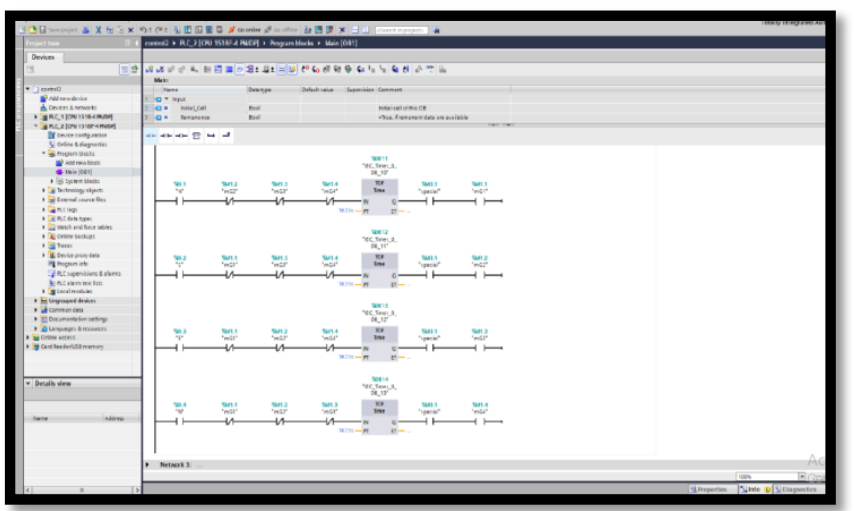

Figure 11

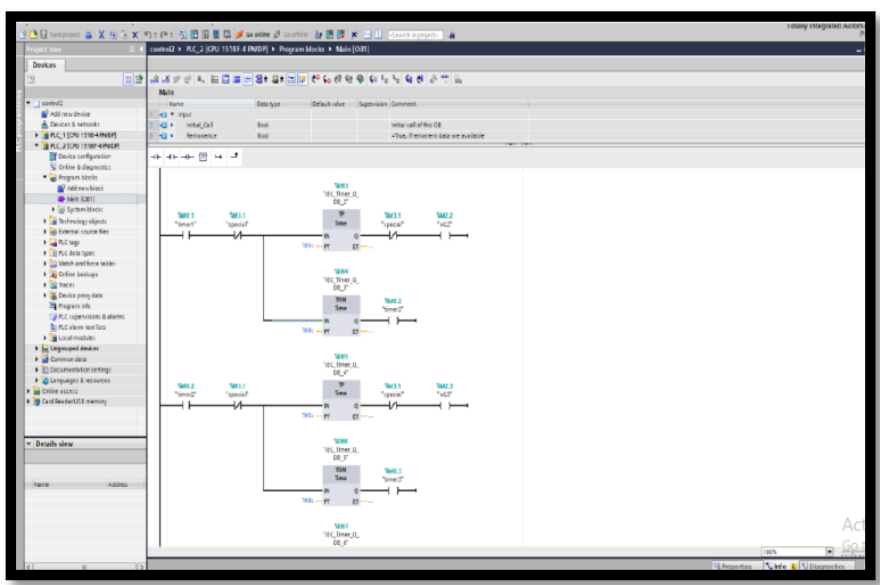

Figure 12 
The outputs of the PLC were connected to relays which were used to switch the intersections between green and red. The traffic lights LEDs were powered by 5V DC from the breadboard Power supply.

\section{Results}

During the normal state, the traffic light operates in its conventional mode as stated in the state table. When the emergency vehicles reach the intersection, the driver presses the push button initiating the emergency scheme. All the emergency lights then switch indicating the presence of the emergency vehicle.The intersection is then cleared. The emergency vehicles lane is then given the right of way.

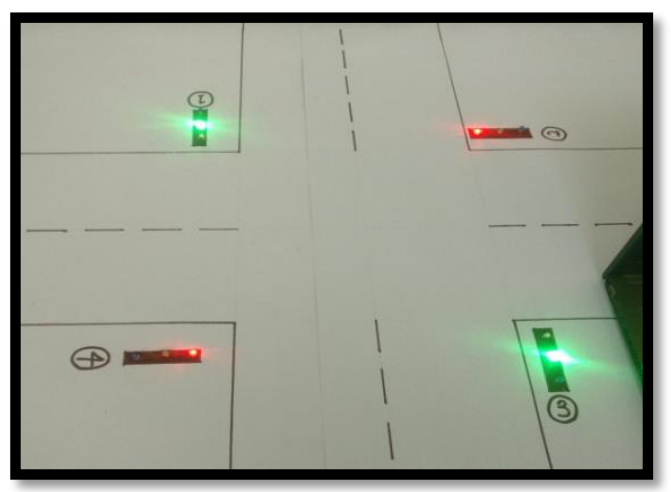

Figure 13: Displays the Normal Operating State of the Traffic Light

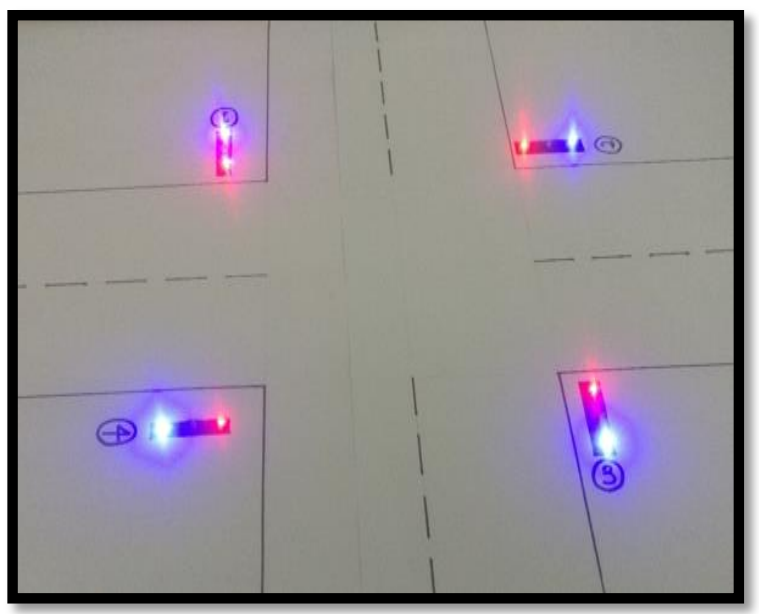

Figure 14: Shows the First Phase of the Emergency State after an RF Signal Has Been Transmitted By An Emergency Vehicle

This state forewarns all the lanes the presence of an emergency vehicle and the timing allows the intersection to clear before pre-emption done on the particular lane.

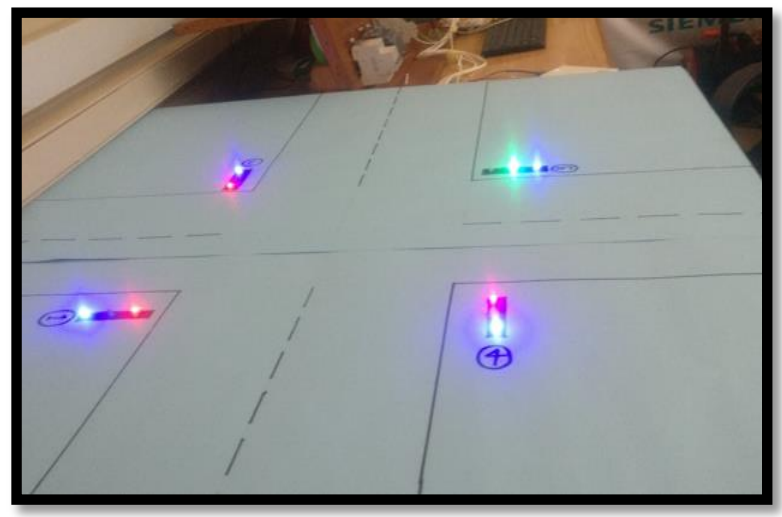

Figure 15: Shows the Second Phase of the Emergency State

After pre-emption, the green light glows to allow quick passage of the emergency vehicle at the intersection. After the vehicle leaves the junction the system returns to its normal mode 


\section{Conclusions}

At the end of the project, we successfully designed and built an emergency vehicle pre-emption system that could;

- Detect the presence of emergency vehicles at an intersection.

- Switch from the normal operation of the traffic light to an emergency mode which offered right of way to the emergency vehicle.

- Resume the normal traffic light operation after the emergency vehicle had passed.

Our system is cost effective. Implementing this system in already existing traffic lights will require just a few modifications. Implementing this system in our transportation system will improve the response time of emergency vehicles while increasing safety of all road users at an intersection during an emergency.

\section{References}

i. Bonner, E. L., \& Faerber, N. A. (1980, September 16). Emergency control system for traffic signals. Google Patents. (US Patent 4,223,295)

ii. Hashim, N., Jaafar, A., Ali, N., Salahuddin, L., Mohamad, N., \& Ibrahim, M. (2013). Traffic light control system for emergency vehicles using radio frequency. IOSR Journal of Engineering (IOSRJEN),3(7), 43\{52.

iii. Kissell, T. E., Karr, S. A., \& Overstreet, R. C. (2003). Laboratory manual to accompany industrial electronics: Applications for programmable controllers, instrumentation and process control, and electrical machines and motor controls. Prentice Hall.

iv. Mitchell, W. L. (1984, April 17). Traffic light control for emergency vehicles. Google Patents. (US Patent 4,443,783)

v. Qi, L., Zhou, M., \& Luan, W. (2015). Emergency traffic-light control system design for intersections subject to accidents. IEEE Transactions on Intelligent Transportation Systems, 17(1), $170\{183$.

vi. Sharma, J., \& Sivani, S. (2014). LAN based traffic light control system with emergency service identification and density based control. International Journal of Engineering Science Invention, 3, $01\{03$.

vii. Zald'ıvar-Colado, A., Tripp-Barba, C., Brito-Rojas, J. A., Aguilar-Calder'on, J. A., Garc'ı́-S'anchez, O. V., Ram'ırezZambada, L., \& Zatarain-Montalvo, J. N. (2017). Management of traffic lights for emergency services. Tehnicki vjesnik, 24(2), 643\{64 